ARTICLE

DOI: $10.1038 / s 41467-018-07197-8$

\title{
A biomimetic nanoreactor for synergistic chemiexcited photodynamic therapy and starvation therapy against tumor metastasis
}

\author{
Zhengze $\mathrm{Yu}^{1}$, Ping Zhou ${ }^{1}$, Wei Pan ${ }^{1}, \mathrm{Na} \mathrm{Li}^{1} \&$ Bo Tang${ }^{1}$
}

Photodynamic therapy (PDT) is ineffective against deeply seated metastatic tumors due to poor penetration of the excitation light. Herein, we developed a biomimetic nanoreactor (bioNR) to achieve synergistic chemiexcited photodynamic-starvation therapy against tumor metastasis. Photosensitizers on the hollow mesoporous silica nanoparticles (HMSNs) are excited by chemical energy in situ of the deep metastatic tumor to generate singlet oxygen $\left({ }^{1} \mathrm{O}_{2}\right)$ for PDT, and glucose oxidase (GOx) catalyzes glucose into hydrogen peroxide $\left(\mathrm{H}_{2} \mathrm{O}_{2}\right)$. Remarkably, this process not only blocks the nutrient supply for starvation therapy but also provides $\mathrm{H}_{2} \mathrm{O}_{2}$ to synergistically enhance PDT. Cancer cell membrane coating endows the nanoparticle with biological properties of homologous adhesion and immune escape. Thus, bio-NRs can effectively convert the glucose into ${ }^{1} \mathrm{O}_{2}$ in metastatic tumors. The excellent therapeutic effects of bio-NRs in vitro and in vivo indicate their great potential for cancer metastasis therapy.

\footnotetext{
${ }^{1}$ College of Chemistry, Chemical Engineering and Materials Science, Key Laboratory of Molecular and Nano Probes, Ministry of Education, Collaborative Innovation Center of Functionalized Probes for Chemical Imaging in Universities of Shandong, Institute of Molecular and Nano Science, Shandong Normal University, Jinan 250014, China. Correspondence and requests for materials should be addressed to N.L. (email: lina@sdnu.edu.cn)

or to B.T. (email: tangb@sdnu.edu.cn)
} 
T he metastatic spread of cancer cells is disastrous for patients and often leads to death ${ }^{1,2}$. As a promising candidate for curing cancer, photodynamic therapy (PDT) has performed well and proven to be effective in many cancers over past decades ${ }^{3-5}$. Recent PDT in the clinic focuses on superficial tumors or lesions that are accessible through endoscopes, such as oral cancer, skin cancer, and esophageal cancer ${ }^{6}$. However, it is frustrating that PDT is almost not useful against cancer metastasis because the low penetration of excitation light makes it impossible to reach deep metastasis sites ${ }^{7-10}$. Although researchers have designed near-infrared light triggered photosensitizers to overcome penetration problems, these photosensitizers still suffer from low efficiency ${ }^{11-13}$. To improve the clinical application of PDT to deeply seated metastases, it is feasible to conduct PDT using chemical energy instead of light excitation $^{14,15}$; this chemical energy can be produced by a reaction between hydrogen peroxide $\left(\mathrm{H}_{2} \mathrm{O}_{2}\right)$ and peroxyoxalate derivatives ${ }^{16,17}$. However, the intracellular $\mathrm{H}_{2} \mathrm{O}_{2}$ concentration is rather low (less than $0.1 \mu \mathrm{M})^{18}$ and cannot generate sufficient chemical energy, severely limiting chemiluminescence resonance energy transfer (CRET)-based PDT. Cancer starvation therapy is another emerging therapeutic method that blocks nutrient supply to suppress tumor growth ${ }^{19-21}$. Considering the essential role of glucose in cancer cell proliferation and metabolism, we chose glucose oxidase (GOx) to consume intracellular glucose through a glucose-involved reaction that catalyzes the conversion of glucose into gluconic acid and $\mathrm{H}_{2} \mathrm{O}_{2}{ }^{22,23}$. Remarkably, this process can not only deplete intracellular glucose for starvation therapy but also increase endogenous $\mathrm{H}_{2} \mathrm{O}_{2}$ levels to generate reactive oxygen species (ROS) for PDT. Thus, chemiexcited PDT combined with starvation therapy is an ideal candidate for treating cancer metastasis.

Furthermore, both the PDT and the oxidation of glucose depend on oxygen $\left(\mathrm{O}_{2}\right)$. The consumption of $\mathrm{O}_{2}$ will greatly affect the production of $\mathrm{H}_{2} \mathrm{O}_{2}$ and the PDT effect. In addition, the hypoxic properties of the tumor environment, especially those in the inner part of the solid tumor, greatly limit the performance of $\mathrm{PDT}^{24-26}$. Consequently, new $\mathrm{O}_{2}$-carrying nanoparticles are expected to enhance the synergistic effects of PDT and starvation therapy. Nanoparticles are traditionally surface functionalized with folic acid, polyethylene glycol, peptides, aptamers, or polymers to improve their tumor-targeting ability $27-31$. However, most of them are still eliminated by the reticuloendothelial system during blood circulation, resulting in low targeting efficiency ${ }^{32,33}$. Cancer cells can perform immune escape and homologous adhesion due to their specific plasma membrane proteins ${ }^{34-37}$. Therefore, biomimetic nanoparticles with cancer cell membranes will greatly improve the delivery efficiency of nanoparticles to tumors.

In the present work, we designed a CRET-based biomimetic nanoreactor (bio-NR) to perform synergistic photodynamicstarvation therapy against tumor metastases by converting glucose into singlet oxygen $\left({ }^{1} \mathrm{O}_{2}\right)$ in cancer cells. Hollow mesoporous silica nanoparticles (HMSNs) are firstly modified with the photosensitizer chlorin e6 (Ce6) and GOx on the surface, followed by co-encapsulating bis[2,4,5-trichloro-6-(pentyloxycarbonyl)phenyl] oxalate (CPPO) and perfluorohexane (PFC) into the cavity of HMSNs, and then coating with cancer cell membrane. Thus, in this bio-NR, Ce6 will be activated by the chemical energy produced from the reaction between $\mathrm{CPPO}$ and intracellular $\mathrm{H}_{2} \mathrm{O}_{2}$ to generate ROS via CRET for PDT with no light excitation. At the same time, the conversion of glucose into $\mathrm{H}_{2} \mathrm{O}_{2}$ will be catalyzed by GOx, which not only consumes nutrients for starvation therapy but also enhances PDT synergistically due to the $\mathrm{H}_{2} \mathrm{O}_{2}$ supply. Furthermore, PFC can carry $\mathrm{O}_{2}$ to modulate the hypoxic environment of the tumor and accelerate the rate of glucose oxidation and ROS generation. In addition, the cancer cell membrane coating confers excellent targeting ability via immune escape and homologous adhesion to the nanoreactor. The structure of the bio-NR and the details of using synergetic PDT and starvation therapy via CRET against cancer metastasis are illustrated in Fig. 1.

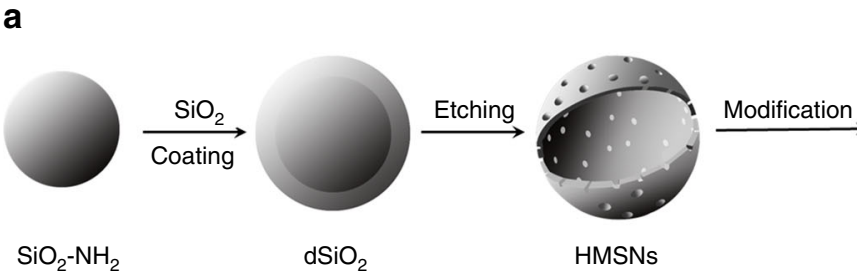

b

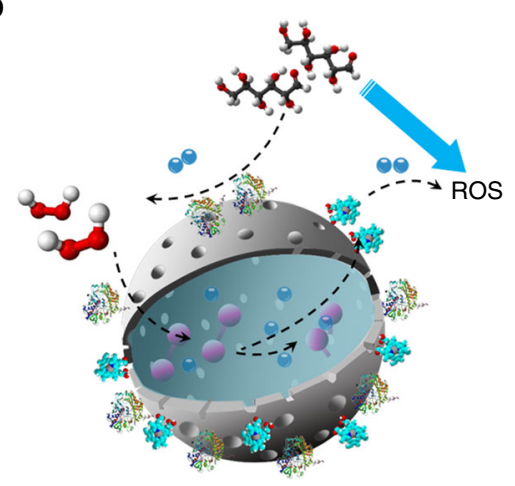

C

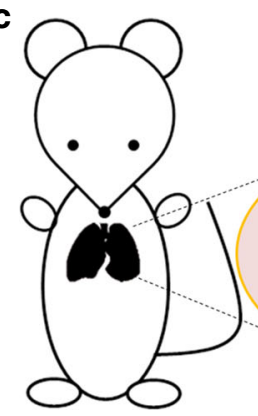

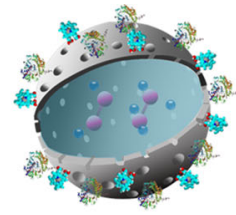

HMSNs-GOx-Ce6 @ CPPO-PFC/O 2

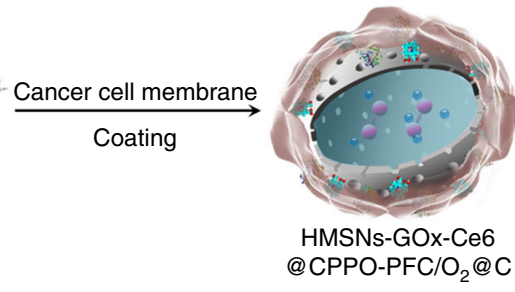

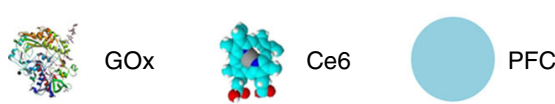
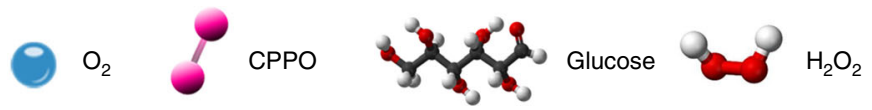

Fig. 1 Schematic illustrations of the process for synthesizing the biomimetic nanoreactor (a), ROS generation based on CRET with glucose consumption with no light excitation (b), and synergetic photodynamic-starvation therapy for metastases (c) 


\section{Results}

Characterization of the bio-NRs. HMSNs were synthesized according to a modified method reported previously ${ }^{38}$. In brief, amino-functionalized silica nanoparticles $\left(\mathrm{SiO}_{2}-\mathrm{NH}_{2}\right)$ were first prepared via a reverse microemulsion method. Then, $\mathrm{SiO}_{2}-\mathrm{NH}_{2}$ was coated with the $\mathrm{SiO}_{2}$ layer to form a core-shell structure $\left(\mathrm{SiO}_{2}-\mathrm{NH}_{2} @ \mathrm{SiO}_{2}\right.$, denoted $\left.\mathrm{dSiO}_{2}\right)$. HMSNs were obtained by selectively etching the $\mathrm{SiO}_{2}$ core using hydrofluoric acid (HF), and the mesoporous structure formed simultaneously. For further decoration and cancer cell membrane coating, HMSNs were modified with amino groups. As shown in the transmission electron microscope (TEM) images, $\mathrm{SiO}_{2}-\mathrm{NH}_{2}$ and $\mathrm{dSiO}$ exhibit uniform spherical morphology with diameters of 50 and $80 \mathrm{~nm}$, respectively (Fig. 2a, b). After the $\mathrm{SiO}_{2}-\mathrm{NH}_{2}$ core had been selectively etched, HMSNs with a homogeneous shell thickness of approximately $10 \mathrm{~nm}$ were present, containing an obvious cavity that can be seen in scanning electron microscope image (Fig. $2 \mathrm{c}$ and Supplementary Fig. 1). The modification of amino groups did not destroy the morphology of HMSNs (Fig. 2d). $\mathrm{N}_{2}$ adsorption-desorption isotherms displayed typical Type IV curves and confirmed the mesoporous structure of HMSNs and HMSNs- $\mathrm{NH}_{2}$. The surface area (via the Brunauer-Emmett-Teller (BET) method) and average pore size (via the Barrett, Joyner, and Halenda (BJH) method) of HMSNs were calculated to be 417.17 $\mathrm{m}^{2} / \mathrm{g}$ and $11.4 \mathrm{~nm}$, and they were decreased to $147.17 \mathrm{~m}^{2} / \mathrm{g}$ and $8.4 \mathrm{~nm}$ after amino modification (Supplementary Fig. 2 and Table 1). The amino content of HMSNs- $\mathrm{NH}_{2}$ was calculated to be $1.12 \mu \mathrm{mol} / \mathrm{mg}$ by thermogravimetric analysis (TGA) (Supplementary Fig. 3). Then, the photosensitizer Ce6 and GOx were anchored on the surface of $\mathrm{HMSNs}-\mathrm{NH}_{2}$ via amide reactions, and $\mathrm{CPPO}$ and PFC were co-encapsulated into the cavity of HMSNs$\mathrm{NH}_{2}$ (HMSNs-GOx-Ce6@PFC-CPPO). The characteristic absorption peaks at 400 and $655 \mathrm{~nm}$ and the fluorescence emission peak at $665 \mathrm{~nm}$ appeared after Ce6 modification, and the Ce6 content was determined to be $0.27 \mu \mathrm{mol} / \mathrm{mg}$ by quantitative fluorescence analysis (Fig. 2f, g and Supplementary Fig. 4). Finally, cancer cell membrane was separated and coated onto the surface of HMSNs-GOx-Ce6@PFC-CPPO to obtain bio-NRs (HMSNs-GOx-Ce6@PFC-CPPO@C). As clearly seen in the TEM image, a lipid layer with a thickness of approximately $10 \mathrm{~nm}$ is present, demonstrating the successful coating with the membrane (Fig. 2e). The hydrodynamic diameter of the different nanoparticles was measured using dynamic light scattering; the diameter increased continuously with each step of the process: $108 \pm$ $15 \mathrm{~nm}$ for $\mathrm{SiO}_{2}-\mathrm{NH}_{2}, 135 \pm 13 \mathrm{~nm}$ for $\mathrm{dSiO}_{2}, 136 \pm 11 \mathrm{~nm}$ for HMSNs, $143 \pm 12 \mathrm{~nm}$ for HMSNs- $\mathrm{NH}_{2}$, and $165 \pm 11 \mathrm{~nm}$ for bioNRs (Fig. 2h). Moreover, the zeta potential values provided further evidence for the successful construction of nanoparticles in each procedure, with a value of $19.0 \pm 1.2 \mathrm{mV}$ for $\mathrm{SiO}_{2}-\mathrm{NH}_{2}$, $-23.8 \pm 1.5 \mathrm{mV}$ for $\mathrm{dSiO}_{2},-19.1 \pm 0.9 \mathrm{mV}$ for HMSNs, $10.6 \pm$ $0.9 \mathrm{mV}$ for $\mathrm{HMSNs}-\mathrm{NH}_{2}$, and $-12.9 \pm 1.2 \mathrm{mV}$ for bio-NRs (Fig. 2i).

In vitro verification of the conversion. The ability of the bioNRs to convert glucose to ${ }^{1} \mathrm{O}_{2}$ via CRET was first verified in vitro. In this process, GOx catalyzed the conversion of intracellular glucose into $\mathrm{H}_{2} \mathrm{O}_{2}$, which further reacted with CPPO to produce chemical energy for the photosensitizer Ce6 to generate ${ }^{1} \mathrm{O}_{2}$. For the detection of $\mathrm{H}_{2} \mathrm{O}_{2}$ produced during the catalytic process, a fluorescence probe that has a specific response to $\mathrm{H}_{2} \mathrm{O}_{2}, \mathrm{Cy}-\mathrm{O}-\mathrm{Eb}$, was employed in fluorescence analysis ${ }^{39}$. As shown in the fluorescence spectra, the intensity of Cy-O-Eb clearly increased when HMSNs-GOx-Ce6 were incubated with glucose $(1 \mathrm{mM})$, indicating $\mathrm{H}_{2} \mathrm{O}_{2}$ generation (Fig. 3a). Furthermore, electron spin resonance (ESR) spectroscopy was also used for the analysis of $\mathrm{H}_{2} \mathrm{O}_{2}$ generation. $\mathrm{H}_{2} \mathrm{O}_{2}$ will convert to hydroxyl radicals $(\cdot \mathrm{OH})$ in the presence of $\mathrm{Fe}^{2+}$ through the Fenton reaction, and these radicals can be captured by a radical scavenger, 2,2,6,6-tetramethylpiperidine (TEMPO) ${ }^{40,41}$. The decreased intensity of the three peaks in the ESR spectra after the addition of glucose demonstrated $\cdot \mathrm{OH}$ generation and further confirmed the production of $\mathrm{H}_{2} \mathrm{O}_{2}$ (Fig. $3 \mathrm{~b}$ ). In addition, the $\mathrm{pH}$ value was monitored during the reaction, and it continually decreased (from 7.28 to 3.78 within $60 \mathrm{~min}$ ) as a result of the production of gluconic acid. These results indicated that GOx remained active after modified on the HMSNs and could catalyze the conversion of glucose into $\mathrm{H}_{2} \mathrm{O}_{2}$ (Fig. $3 \mathrm{c}$ ). Then, $\mathrm{H}_{2} \mathrm{O}_{2}$ reacts with CPPO in the cavity of HMSNs to form a high-energy intermediate and consequently excite $\mathrm{Ce} 6$ to generate ${ }^{1} \mathrm{O}_{2}$, which was detected using
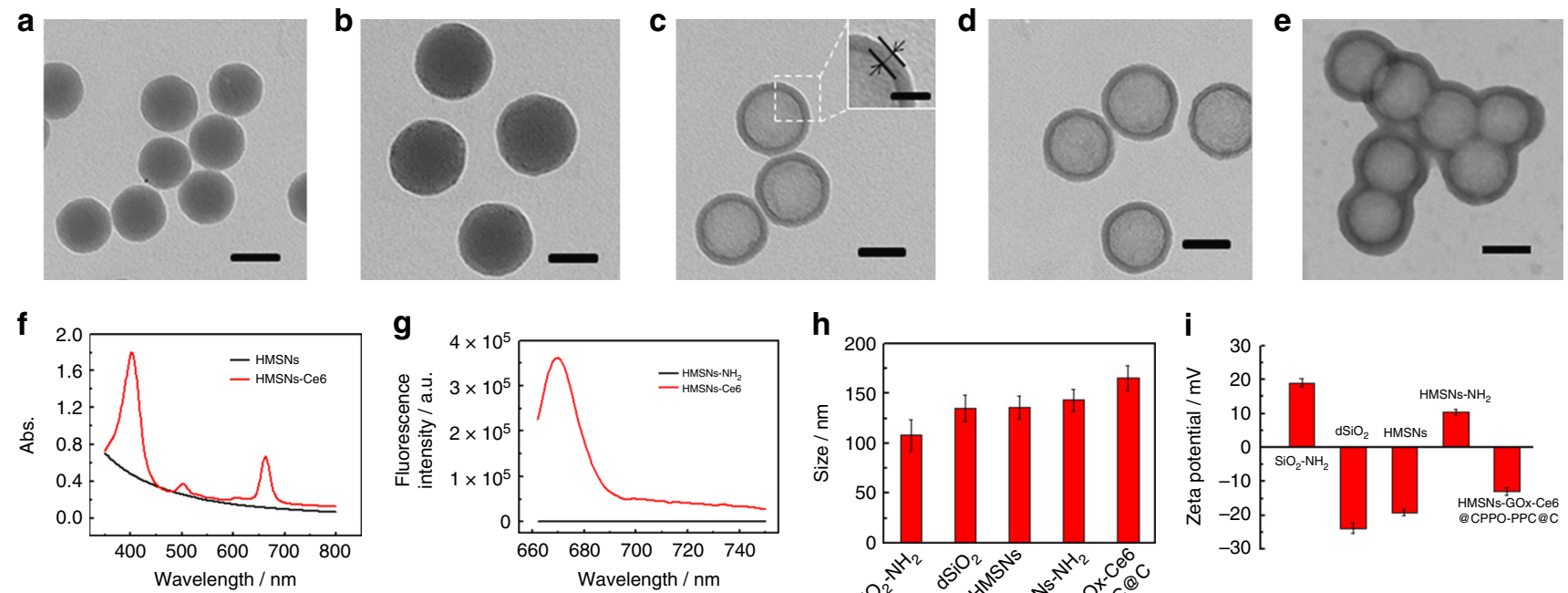

$\mathbf{h}$

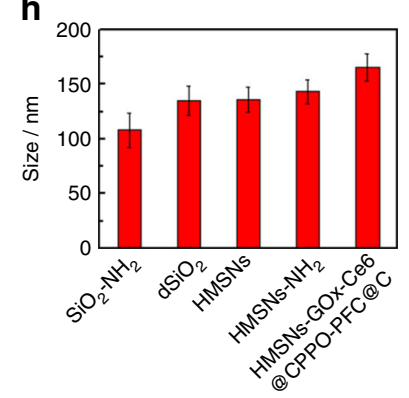

i

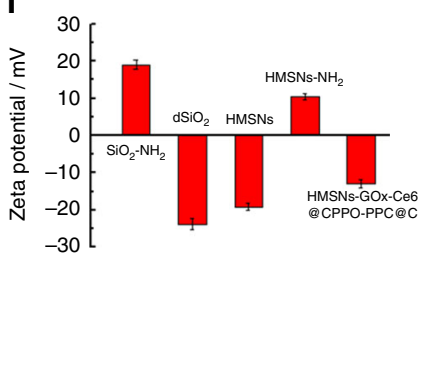

Fig. 2 Characterization of the biomimetic nanoreactor. TEM images of $\mathrm{SiO}_{2}-\mathrm{NH}_{2}(\mathbf{a}), \mathrm{dSiO} 2(\mathbf{b}), \mathrm{HMSNs}(\mathbf{c}), \mathrm{HMSNs}-\mathrm{NH} \mathrm{H}_{2}$ (d), and HMSNs-GOx-

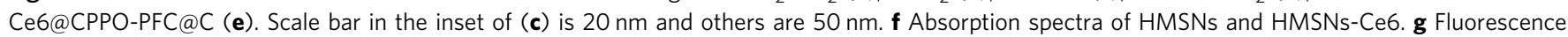
spectra of HMSNs and HMSNs-Ce6. Hydrodynamic size distributions (h) and zeta potentials (i) of the nanoparticles 

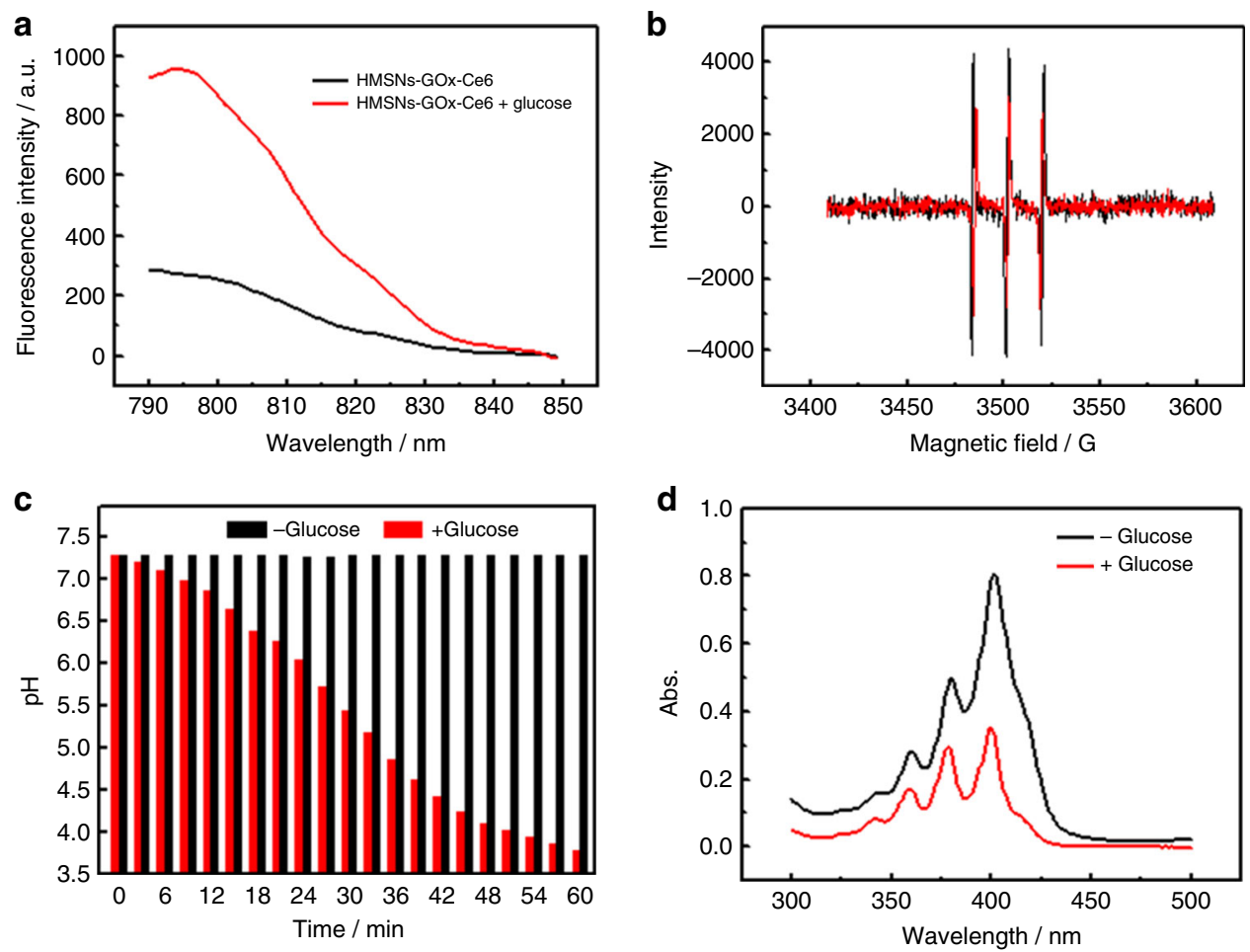

Fig. 3 Verification of $\mathrm{H}_{2} \mathrm{O}_{2}$ and ${ }^{1} \mathrm{O}_{2}$ generation in vitro. a Fluorescence spectra of Cy-O-Eb probe incubated with HMSNs-GOx-Ce6 in the presence or absence of $1 \mathrm{mM}$ glucose. b ESR spectra of TEMPO after incubation with HMSNs-GOx-Ce6 and Fe ${ }^{2+}$ in the presence (red) or absence (black) of glucose. c The $\mathrm{pH}$ values of HMSNs-GOx-Ce6 solution in the presence or absence of $1 \mathrm{mM}$ glucose. $\mathbf{d}$ Absorption spectra of ABMD incubated with HMSNs-GOxCe6@CPPO-PFC/O $/ \mathrm{O}_{2}$ in the absence or presence of $1 \mathrm{mM}$ glucose

UV-visible (UV-vis) spectrum light by measuring the absorption changes of 9,10-anthracenediyl-bis(methylene)dimalonic acid (ABMD). As shown in Fig. 3d, the absorption intensity decreased sharply when HMSNs-GOx-Ce6@CPPO was incubated with glucose, suggesting the generation of ${ }^{1} \mathrm{O}_{2}$ from glucose.

Intracellular therapeutic effects. Intracellular experiments were carried out to evaluate the bio-NRs in cancer metastasis therapy. Notably, to maximize the simulation of the hypoxic tumor microenvironment, all the experiments were carried out under hypoxic conditions. The ability of the bio-NRs to modulate the hypoxic tumor microenvironment was assessed. Because of the cavity in the HMSNs, bio-NRs can act as a carrier for PFC loading, which can absorb large amounts of $\mathrm{O}_{2}$ due to the van der Waals interactions between PFC and $\mathrm{O}_{2}$. Bio-NRs can thus release $\mathrm{O}_{2}$ in the oxygen-deficient environment. When cells are in the hypoxic environment, they overexpress hypoxia-inducible factor $1 \alpha(\mathrm{HIF}-1 \alpha)^{42}$, which can be detected by immunofluorescent staining using confocal laser scanning microscopy (CLSM) in mouse melanoma cells (B16-F10). As shown in Fig. $4 \mathrm{a}$, a bright green fluorescence signal was observed when B16-F10 cells were treated with HMSNs@PFC@C, while the fluorescence intensity was rather low if HMSNs@PFC@C were pretreated with $\mathrm{O}_{2}$ (HMSNs@PFC/O $\left.\mathrm{H}_{2} @ \mathrm{C}\right)$. The results show that HIF-1a level was low in B16-F10 treated with HMSNs@PFC/ $\mathrm{O}_{2} @ \mathrm{C}$, indicating that the nanoparticles carried $\mathrm{O}_{2}$ and modulated the hypoxic tumor microenvironment. Intracellular conversion of glucose into $\mathrm{H}_{2} \mathrm{O}_{2}$ by the bio-NRs in B16-F10 cells was then verified using CLSM. Confocal images show a distinct red signal of the fluorescence probe $\mathrm{Cy}-\mathrm{O}-\mathrm{Eb}$ in B16-F10 cells incubated with HMSNs-GOx@PFC/O $\mathrm{O}_{2} @ \mathrm{C}$ or HMSNs-GOx@PFC@C, which demonstrated that bio-NRs catalyzed the conversion of glucose into $\mathrm{H}_{2} \mathrm{O}_{2}$. Moreover, the former materials showed higher fluorescence intensity than the latter due to the $\mathrm{O}_{2}$ that it carried, indicating a larger amount of $\mathrm{H}_{2} \mathrm{O}_{2}$ generation and revealing $\mathrm{O}_{2}$ to be a key factor (Fig. 4b). We also tested the synergetic photodynamic-starvation therapeutic effect of the bio-NRs against cancer cells with no light excitation via an MTT (3(4,5-dimethylthiazol-2-yl)-2,5-diphenyltetrazolium bromide) assay. Supplementary Fig. 5 displays the viability of cells receiving different treatments. The data showed that PDT or starvation therapy alone resulted in higher cell viability $(73.8 \%$ and $62.0 \%$, respectively) than the combination of therapies. Moreover, the viability of cells incubated with bio-NRs lacking $\mathrm{O}_{2}$ reached $57.1 \%$, confirming the important role of $\mathrm{O}_{2}$. Remarkably, the viability of B16-F10 cells was reduced to $20.7 \%$ when they were incubated with bio-NRs, indicating their excellent therapeutic effects. What's more, the effect of starvation therapy alone and PDT alone under anaerobic conditions was evaluated and the cell viabilities were $83.7 \%$ and $89.6 \%$, which further demonstrated the key role of $\mathrm{O}_{2}$ for both starvation therapy and PDT (Supplementary Fig. 6).

Homologous adhesion and immune escape in vivo. As the characteristics of the cancer cell membrane depend on its membrane proteins, we identified five major proteins associated with cell invasion and metastasis (CD44, CD47, E-cadherin, EpCAM, and Tissue factor) using western blot ${ }^{43-45}$. The data showed that these proteins were not destroyed during the extraction of the cell membrane and its coating on the nanoparticles (Fig. 4c). Then, the targeting ability of bio-NRs was evaluated via in vivo imaging. B16-F10 cells were injected intravenously into $\mathrm{BalB} / \mathrm{C}$ mice to construct a lung metastasis mouse model, which was followed by intravenous injection with HMSNs-Ce6 or HMSNs-Ce6@C. At 24 h post injection, the mice were killed and dissected for in vivo imaging. From in vivo 
a
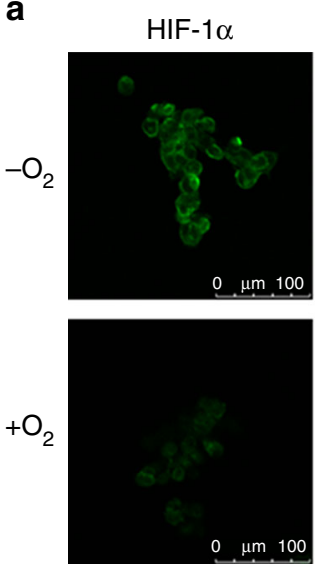

d

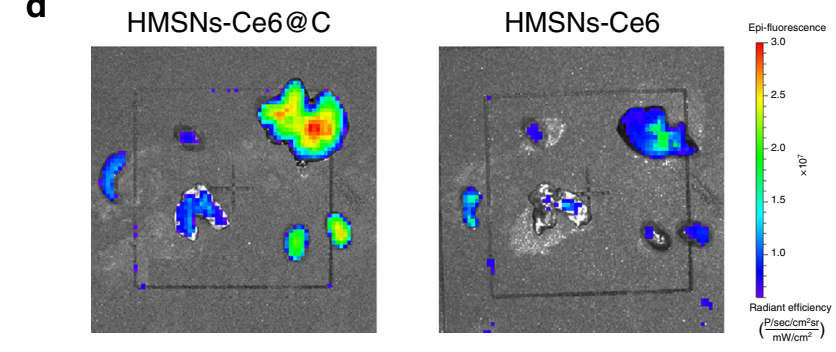

Bright field
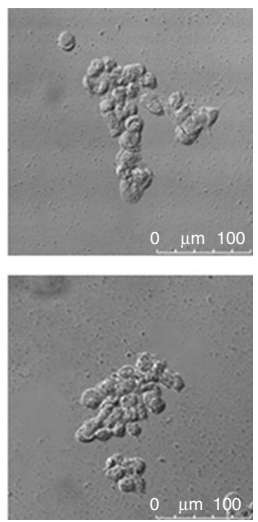

b
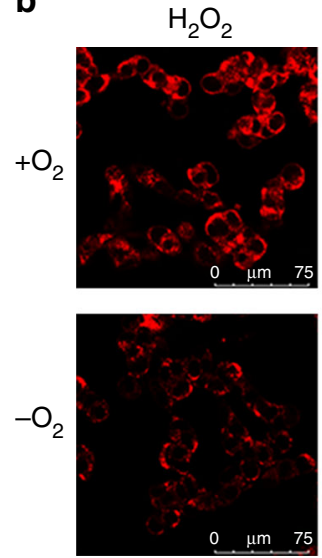

Bright field
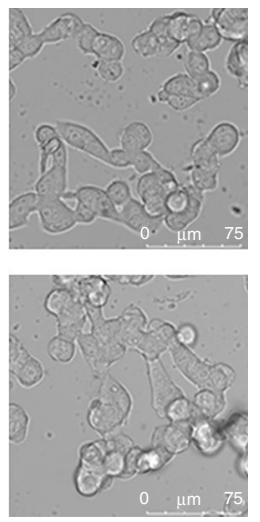

C

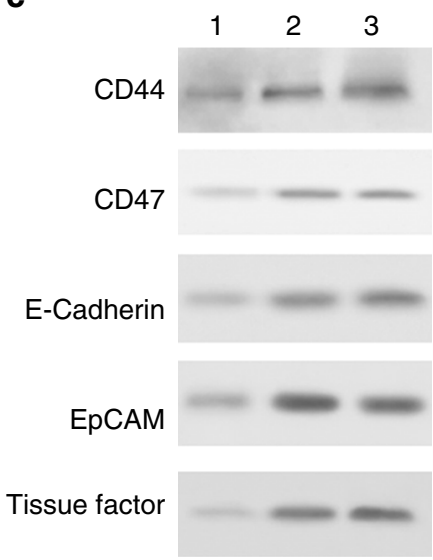

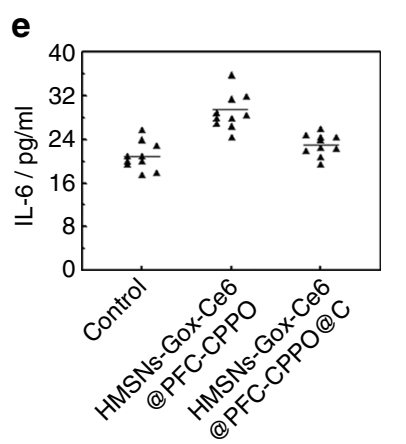

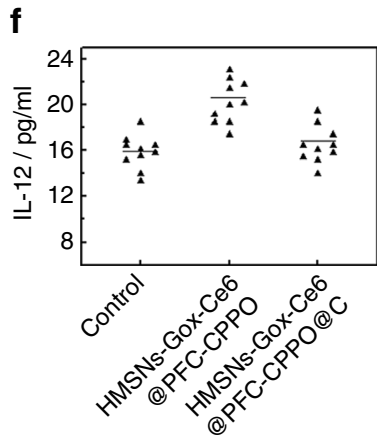

Fig. 4 a Immunofluorescent staining images of HIF-1 $\alpha$ in B16-F10 pre-incubated with HMSNs@PFC@C (top) or HMSNs@PFC/O2@C (bottom) in a hypoxic environment. b CLSM images of B16-F10 cells incubated with Cy-O-Eb and HMSNs-GOx@PFC/O $2 @ C$ (top) or HMSNs-GOx@PFC@C (bottom). cWestern blots of CD44, CD47, E-Cadherin, EpCAM, and tissue factor. Column 1, B16-F10 cells; column 2, B16-F10 cell membranes; column 3, HMSNs@C. d In vivo imaging of lung with metastatic tumor at $24 \mathrm{~h}$ post injection of HMSNs-Ce6@C (left) or HMSNs-Ce6 (right). White arrows point to the lungs. Enzyme-linked immunosorbent assay (ELISA) analysis of IL-6 (e) and IL-12 (f) after the mice were injected with HMSNs-GOx-Ce6@CPPO-PFC@C or HMSNs-GOxCe6@CPPO-PFC

images, we see that lung in the mice treated with HMSNs-Ce6@C exhibited a brighter signal than lung in control mice, indicating the good targeting efficiency after the cancer cell membrane coating (Fig. 4d). Moreover, the fluorescence intensity in the liver and kidney of mice treated with HMSNs-Ce6 was weaker than that of mice treated with HMSNs-Ce6@C, which was mainly due to rapid metabolism by the immune system. These results confirm that nanoparticles can perform homologous adhesion and immune escape. The targeting ability of cancer cell membranecoated nanoparticles was also assessed using inductively coupled plasma atomic emission spectrometry (ICP-AES) (Supplementary Fig. 7). Data showed that the targeting effect of cell membranecoated nanoparticles was much better than that of the nanoparticles without the membrane coating. What's more, large amounts of nanoparticles without membrane accumulated in liver and spleen, indicating the rapid clearance. Interleukin-6 (IL6) and interleukin-12 (IL-12) are secreted by immune cells to stimulate the immune response, and they are often used for the identification of immune activation ${ }^{46,47}$. Therefore, IL-6 and IL12 were monitored to verify the immune escape of the designed bio-NRs. Figure 4e, f display the expression levels of IL-6 and IL12 in the blood after mice were injected with bio-NRs or HMSNsGOx-Ce6@PFC-CPPO. We found that the blood concentration of IL- 6 and IL-12 in mice treated with bio-NRs was similar to that of mice without treatment, indicating that bio-NRs indeed achieve immune escape. However, nanoparticles without the membrane coating caused an immune response, reflected by a clear increase in the blood concentration of IL-6 and IL-12; as a result, these nanoparticles would be cleared from the body faster than the membrane-coated nanoparticles (Supplementary Fig. 8).

Synergetic therapy for a lung metastatic tumor. The synergetic photodynamic-starvation therapeutic effect of bio-NRs for metastatic tumors was evaluated in vivo. Figure 5a illustrates the details of the therapeutic process. Mice with lung metastatic tumors were injected intravenously with phosphate-buffered saline (PBS), HMSNs-GOx@CPPO-PFC/O ${ }_{2} @ \mathrm{C}, \mathrm{HMSN}$ Ce6@CPPO-PFC/O @@, HMSNs-GOx-Ce6@CPPO-PFC@C, and HMSNs-GOx-Ce6@CPPO-PFC/O $\mathrm{O}_{2} @ \mathrm{C}$ at a dose of $40 \mathrm{mg} / \mathrm{kg}$. After 14 days of treatment, we dissected the lungs and evaluated the therapeutic effect. From the photographs in Fig. 5b, we can see that lung metastatic tumors completely disappeared when the mice received the bio-NRs, demonstrating an excellent therapeutic effect. However, in the control groups, distinct B16-F10 metastatic tumors were observed, and the metastatic rate was rather high (Fig. 5c). Furthermore, the body weight of mice treated with the bio-NRs did not decrease with time, while there were different degrees of weight loss in the other groups due to rapid growth of the metastatic tumors (Fig. 5d). In addition, bioNR-treated mice exhibited a survival rate of $100 \%$, which further confirmed the high therapeutic efficiency of bio-NRs (Fig. 5e). In addition, hematoxylin and eosin (H\&E) staining was employed to verify the synergetic photodynamic-starvation therapeutic effect of bio-NRs. As shown in Fig. 5f, no appreciable lung metastatic 
a
Intravenous injection
1. Metastasis formation
of B16-F10 cells
2. Intravenous injection of
Record body weight
1. Sacrifice the mice and dissect
biomimetic nanoreactor change every other day
2. Observe and imaging

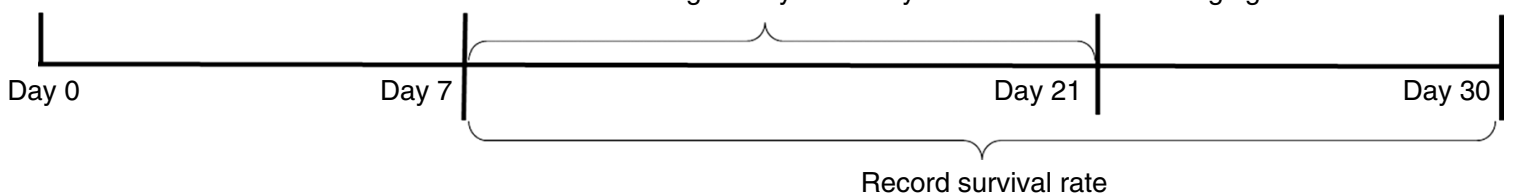

b

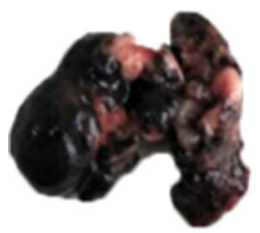

C

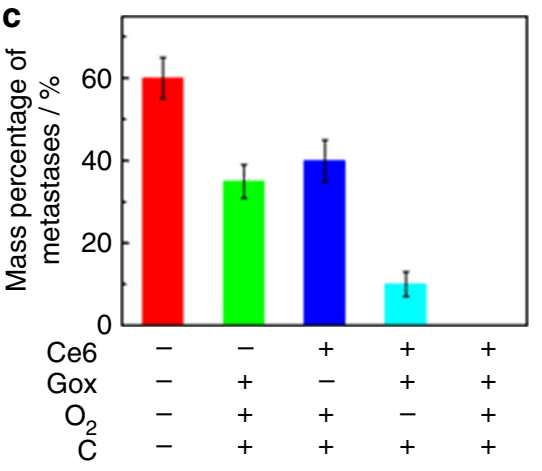

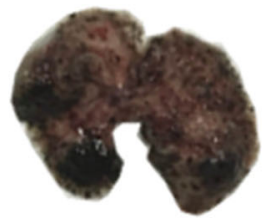

d

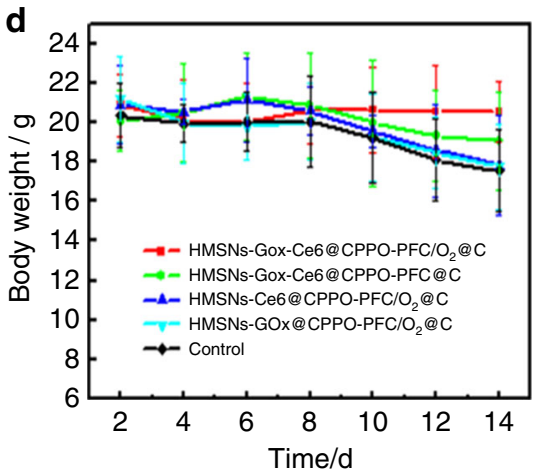

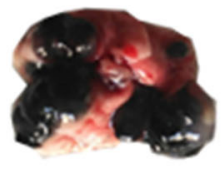

Time/d
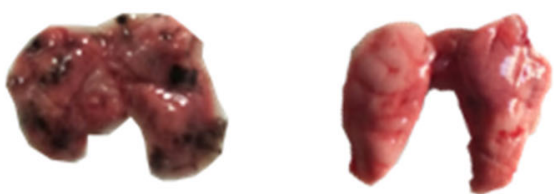

e

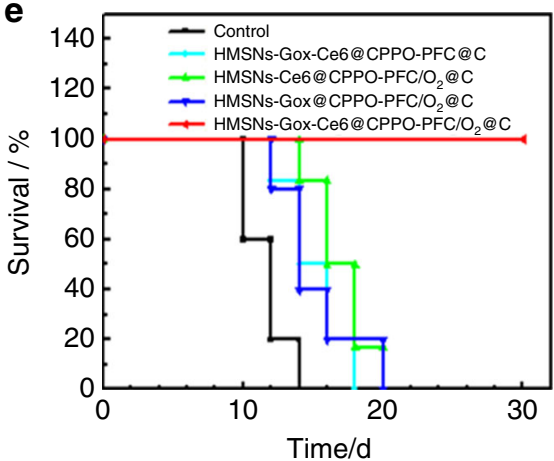

f

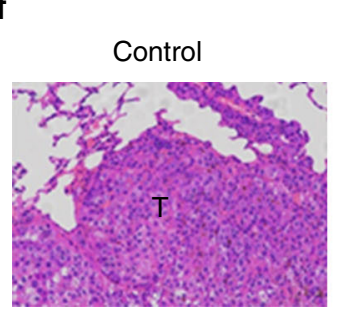

HMSNs-GOx@ CPPO-PFC/O $\mathrm{O}_{2} @ \mathrm{C}$

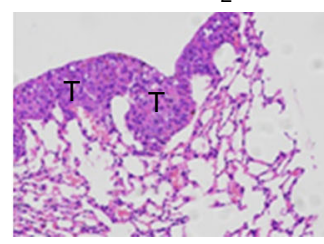

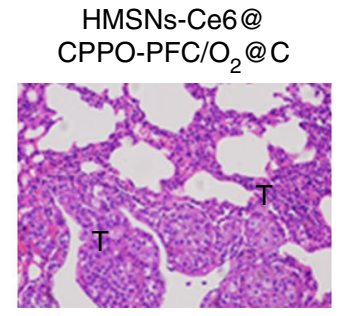

HMSNs-Ce6@ CPPO-PFC/O 20

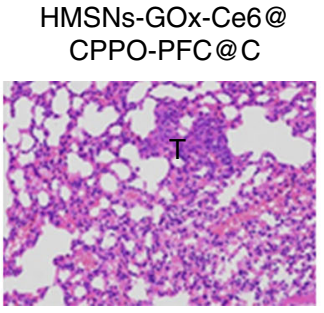

HMSNs-GOx-Ce6@ CPPO-PFC/O $@$ @C

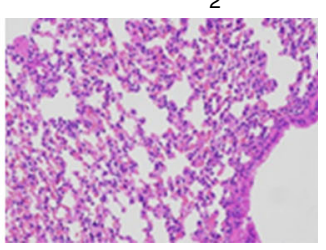

Fig. 5 Therapeutic effects of the bio-NRs in vivo. a Schematic illustration of the in vivo therapeutic process. b Macroscopic images of lungs receiving different treatments. From left to right: control,HMSNs-GOx@CPPO-PFC/O $2 @ C, H M S N s-C e 6 @ C P P O-P F C / O_{2} @ C, H M S N s-G O x-C e 6 @ C P P O-P F C @ C$, and HMSNs-GOx-Ce6@CPPO-PFC/O $@$ @. c The mass percentage of metastases. Mass percentage of metastases/\%=weight of metastatic tumors/ weight of normal lung. $\mathbf{d}$ Body weight curves of mice with metastatic tumors in each group. e Survival rates for each group after receiving treatments. $\mathbf{f} \mathrm{H} \& \mathrm{E}$ staining of lung-bearing metastatic tumors after different treatments $(\times 200$, scale bars are $100 \mu \mathrm{m}) . \mathrm{T}=$ tumor

tissues were noticed in mice with bio-NRs, indicating the complete removal of metastases. In contrast, the metastatic tumors still existed in all the other groups. The histological effect of the nanoparticles on four major organs (liver, spleen, kidney, and heart) was monitored. H\&E stain images showed that there is mild liver injury in mice with nanoparticles that lack the cancer cell membrane coating. This injury occurs because the liver is an immune organ that responds to gather and clear foreign nanoparticles. Nanoparticles without the cancer cell membrane coating cause an immune response and accumulate in the liver. However, cancer cell membrane-coated nanoparticles had no influence on the liver and other organs, suggesting their good biocompatibility (Supplementary Fig. 9).

\section{Discussion}

For the treatment of deep metastatic tumors, an efficient therapeutic method was developed using a bio-NR that conducts synergetic chemiexcited PDT and starvation therapy. To make this nanoreactor, HMSNs were surface-modified with Ce6 and GOx and encapsulated with CPPO in the cavity. Thus, Ce6 could be activated by chemical energy produced from the reaction between CPPO and intracellular $\mathrm{H}_{2} \mathrm{O}_{2}$ via CRET, achieving PDT with no light excitation. Remarkably, GOx can catalyze the conversion of intracellular glucose for synergetic starvation therapy, and a reaction product, $\mathrm{H}_{2} \mathrm{O}_{2}$, will further react with $\mathrm{CPPO}$ to enhance the PDT. Moreover, PFC was co-loaded in the cavity of HMSNs for $\mathrm{O}_{2}$ supply, which solved the problem of $\mathrm{O}_{2}$ lacking and contributed greatly to glucose oxidation and ROS generation. Disguising the nanoparticles with the cancer cell membrane effectively promoted the targeting ability and delivery efficiency of the nanoparticles. The experimental results showed that the PDT therapeutic effect for cancer cells and tumor metastasis was greatly improved after PDT was combined with starvation therapy, indicating that the designed bio-NR has great potential in clinical applications to cancer metastases. We 
anticipate that this approach will provide new sight and reference value for clinical cancer metastasis therapy.

\section{Methods}

Materials and reagents. 1-Hexanol, cyclohexane, Triton X-100, tetraethyl orthosilicate (TEOS), HF (40\%), tetrahydrofuran (THF), 1-(3-diaminopropyl)-3ethylcarbodiimide hydrochloride (EDC), and N-hydroxysuccinimide (NHS) were purchased from Alfa Aesar Chemical Ltd. (Tianjin, China); (3-aminopropyl)triethoxysilane (APTES) and CPPO were purchased from Heowns Biochemical Technology Co., Ltd.; Ce6 was purchased from Frontier Scientific Co, Ltd. USA.; TEMPO, protease inhibitors, PFCs, and GOx were purchased from Sigma-Aldrich. Mouse skin melanoma (B16-F10) was purchased from Shanghai Aoluo Biotechnology Co., Ltd. The water used was Mill-Q secondary ultrapure water (18.2 $\mathrm{M} \Omega / \mathrm{cm}$ ). The chemical reagents used in the experiment were of analytical grade and used without purification.

Synthesis of $\mathbf{S i O}_{\mathbf{2}}-\mathrm{NH}_{\mathbf{2}}$. $\mathrm{SiO}_{2}-\mathrm{NH}_{2}$ nanoparticles were prepared by a reverse-phase microemulsion method with some modifications. First, $5.3 \mathrm{~mL}$ of Triton X-100, $22.5 \mathrm{~mL}$ of cyclohexane, $5.4 \mathrm{~mL}$ of $\mathrm{n}$-hexanol, and $1 \mathrm{~mL}$ of water were added to a round bottom flask and followed with $750 \mu \mathrm{L}$ of ammonium hydroxide. After 30 min of stirring, $0.5 \mathrm{~mL}$ of TEOS and $0.1 \mathrm{~mL}$ of APTES were added to the above solution under vigorous stirring, and the reaction proceeded for $12 \mathrm{~h} . \mathrm{SiO}_{2}-\mathrm{NH}_{2}$ was obtained by centrifugation $(10,000 \mathrm{rpm}, 10 \mathrm{~min})$ and washed with absolute ethyl alcohol three times.

Synthesis of $\mathbf{d S i O}_{2}$. The as-synthesized $\mathrm{SiO}_{2}-\mathrm{NH}_{2}$ was dispersed in $60 \mathrm{~mL}$ of absolute ethyl alcohol. Then, $10 \mathrm{~mL}$ of water and $10 \mathrm{~mL}$ of ammonium hydroxide were added, and the solution was stirred for $30 \mathrm{~min}$. Subsequently, a solution containing $0.3 \mathrm{~mL}$ of TEOS and $9.7 \mathrm{~mL}$ of absolute ethyl alcohol was dropwise added, and the reaction proceeded for $3 \mathrm{~h}$ under stirring. The precipitate, $\mathrm{dSiO}_{2}$, was centrifuged $(10,000 \mathrm{rpm}, 10 \mathrm{~min})$ and washed with absolute ethyl alcohol and water three times. Finally, $\mathrm{dSiO}_{2}$ was dispersed in $80 \mathrm{~mL}$ of water for further use.

Synthesis of HMSNs. HMSNs were prepared using a HF etching method. Then, $150 \mu \mathrm{L}$ of $\mathrm{HF}$ solution $(4 \%, \mathrm{w} \%)$ was added to $10 \mathrm{~mL}$ of as-synthesized $\mathrm{dSiO}_{2}$. After vigorous stirring for $6 \mathrm{~min}$, the solution was centrifuged immediately $(10,000 \mathrm{rpm}$, $10 \mathrm{~min}$ ), and the precipitate was washed with absolute ethyl alcohol and water twice. The obtained HMSNs were dispersed in absolute ethyl alcohol.

Synthesis of HMSNs- $\mathbf{N H}_{2}$. The HMSNs prepared above were dissolved in $40 \mathrm{~mL}$ of anhydrous ethanol, and then $16 \mathrm{~mL}$ of water and $400 \mu \mathrm{L}$ of ammonia were added. After mixing, $10 \mu \mathrm{L}$ of APTES was added to the above solution. After stirring overnight at room temperature, the mixture was centrifuged $(10,000 \mathrm{rpm}$, $10 \mathrm{~min}$ ), and the precipitate was washed twice with ethanol and water. Finally, HMSNs- $\mathrm{NH}_{2}$ was dispersed in $10 \mathrm{~mL}$ of $\mathrm{PBS}(\mathrm{pH}=7.4,0.01 \mathrm{M})$. The amino groups in HMSNs- $\mathrm{NH}_{2}$ were quantified by TGA.

Synthesis of HMSNs-GOx-Ce6. HMSNs-Gox-Ce6 was obtained through the amide-forming reaction between the carboxyl groups of GOx and Ce6 and the amino groups of HMSNs- $\mathrm{NH}_{2}$. EDC $(95 \mathrm{mg})$ and NHS $(57 \mathrm{mg})$ were mixed with GOx (4 mg), and EDC (19.7 mg) and NHS (11.5 mg) were mixed with Ce6 (12 mg) in the dark for $30 \mathrm{~min}$ to activate carboxyl groups. The activated GOx and Ce6 were then added to the solution of HMSNs- $\mathrm{NH}_{2}$ under gentle stirring for $24 \mathrm{~h}$. The product HMSNs-GOx-Ce6 were obtained by centrifugation (10,000 rpm, $10 \mathrm{~min})$ and washed three times with PBS buffer to remove unreacted GOx and Ce6.

Synthesis of HMSNs-GOx-Ce6@CPPO-PFC/O 2 . A solution containing $30 \mathrm{mg}$ of HMSNs-Gox-Ce6 was added to a single-necked round-bottomed flask, and the solvent was evaporated by vacuum pump. Then, $1 \mathrm{~mL}$ of THF solution containing $15 \mathrm{mg}$ of CPPO was added, and the mixture was sonicated for $2 \mathrm{~min}$ to allow the CPPO to enter the HMSN cavity. Subsequently, THF was removed by vacuum to obtain HMSNs-Gox-Ce6@CPPO. Then, 300 $\mu \mathrm{L}$ of perfluorohexane (PFC) solution was added to the powder, and the mixture was sonicated in ice water for $1 \mathrm{~min}$. The redundant solvent was evaporated by vacuum. Finally, the materials were dissolved in a suitable amount of PBS ( $\mathrm{pH}=7.4,0.01 \mathrm{M})$. HMSNs-Gox-Ce6@CPPO-PFC that had been dispersed in PBS were stored in an oxygen chamber $\left(\mathrm{O}_{2}\right.$ flow rate $=$ $5 \mathrm{~L} / \mathrm{min}$ ) for $10 \mathrm{~min}$ to achieve oxygen saturation (HMSNs-GOx-Ce6@CPPO-PFC/ $\mathrm{O}_{2}$ ).

Cell culture. The B16-F10 cells used in the experiments were treated with highglucose Dulbecco's modified Eagle's medium containing 10\% fetal bovine serum and $1 \% 100 \mathrm{U} / \mathrm{mL}$ penicillin/streptomycin. Cells were incubated at $37^{\circ} \mathrm{C}$ in a humidified atmosphere with $5 \% \mathrm{CO}_{2}$. Anaerobic culture conditions were $5 \% \mathrm{CO}_{2}$, $1 \% \mathrm{O}_{2}$, and $94 \% \mathrm{~N}_{2}$ at $37^{\circ} \mathrm{C}$.
Synthesis of the bio-NRs. B16-F10 cells were first suspended and centrifuged $(1000 \mathrm{rpm}, 3 \mathrm{~min})$. The cells were washed twice with Tris buffer $(\mathrm{pH}=7.4)$ and then resuspended in Tris buffer with $1 \%$ protease inhibitor. Subsequently, the cells were disrupted with a homogenizer in an ice-water bath. Membrane fragments were obtained by differential centrifugation. Membrane fragments were then mixed with HMSNs-GOx-Ce6@CPPO-PFC in an ice-water bath, and the solution was stirred for $24 \mathrm{~h}$. After centrifugation (10,000 rpm, $10 \mathrm{~min})$, the precipitate was separated and redispersed in PBS buffer.

Verification of the generation of $\mathbf{H}_{\mathbf{2}} \mathbf{O}_{\mathbf{2}}$ and $\mathbf{1}_{\mathbf{2}}$ in vitro. (1) $\mathrm{H}_{2} \mathrm{O}_{2}: \mathrm{A} \mathrm{H}_{2} \mathrm{O}_{2}$ specific molecular probe, $\mathrm{Cy}-\mathrm{O}-\mathrm{Eb}$, was employed for the detection of $\mathrm{H}_{2} \mathrm{O}_{2}$ via fluorescence analysis. Glucose $(1 \mathrm{mg} / \mathrm{mL})$ was added to a HMSNs-GOx-Ce6 $(2 \mathrm{mg} /$ $\mathrm{mL}$ ) solution containing $\mathrm{Cy}-\mathrm{O}-\mathrm{Eb}(10 \mu \mathrm{M})$ at $37^{\circ} \mathrm{C}$, which was then incubated for $12 \mathrm{~h}$. After centrifugation, the fluorescence of the supernate was measured $\left(\lambda_{\mathrm{ex}}=\right.$ $780 \mathrm{~nm}) . \mathrm{H}_{2} \mathrm{O}_{2}$ generation was also verified via ESR by employing the radical scavenger TEMPO. Glucose $(1 \mathrm{mg} / \mathrm{mL})$ was added to a HMSNs-GOx-Ce6 $(2 \mathrm{mg} /$ $\mathrm{mL}$ ) solution containing TEMPO and a small amount of $\mathrm{Fe}^{2+}$ at $37^{\circ} \mathrm{C}$, which was then incubated for $12 \mathrm{~h}$. After centrifugation, the supernate was analyzed via ESR. Glucose was not added to the control groups. (2) ${ }^{1} \mathrm{O}_{2}$ : The generation of ${ }^{1} \mathrm{O}_{2}$ was verified by employing the ${ }^{1} \mathrm{O}_{2}$-specific molecular probe ABMD and analyzing the UV-vis spectra. Glucose $(1 \mathrm{mg} / \mathrm{mL})$ was added to the HMSNs-GOx-Ce6 $(2 \mathrm{mg} /$ $\mathrm{mL}$ ) solution containing $\mathrm{ABMD}(0.1 \mathrm{mM})$ and then incubated at $37^{\circ} \mathrm{C}$ for $12 \mathrm{~h}$, at which point the absorption of ABMD was measured. Glucose was not added to the control groups.

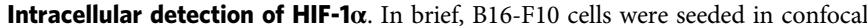
dishes for $24 \mathrm{~h}$. Then, the cells were incubated with HMSNs@PFC@C or HMSNs@PFC/O $@$ @C $(0.2 \mathrm{mg} / \mathrm{mL})$. After further incubation in anaerobic conditions for $4 \mathrm{~h}$, the cells were washed and fixed with precooled $4 \%$ paraformaldehyde at room temperature for $20 \mathrm{~min}$; they were then treated with primary antibody and enhanced secondary antibody for $1 \mathrm{~h}$, respectively. Finally, the cells were washed with PBS three times before confocal microscopy experiments $\left(\lambda_{\mathrm{ex}}=488 \mathrm{~nm}, \lambda_{\mathrm{em}}\right.$ $=500-550 \mathrm{~nm}$ ).

Intracellular detection of $\mathbf{H}_{\mathbf{2}} \mathbf{O}_{\mathbf{2}}$. B16-F10 cells were seeded in confocal dishes for 24 h. Then, the cells were incubated with HMSNs-GOx@PFC@C or HMSNs-GOx@PFC/ $\mathrm{O}_{2} @ \mathrm{C}(0.2 \mathrm{mg} / \mathrm{mL})$ in anaerobic conditions for $24 \mathrm{~h}$. Cy-O-Eb was added, and the cells were further cultured in anaerobic conditions for $10 \mathrm{~min}$ before confocal microscopy experiments. Confocal images were captured with excitation at $633 \mathrm{~nm}$.

MTT assays. (1) B16-F10 cells were incubated in 96-well plates and cultured for $24 \mathrm{~h}$ under anaerobic conditions. The cells were divided into five groups: control,

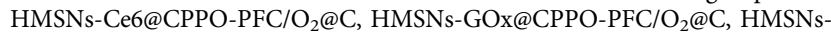
GOx-Ce6@CPPO-PFC@C, and HMSNs-GOx-Ce6@CPPO-PFC/O $@$ @ groups.

The concentration of the nanoparticles was $0.2 \mathrm{mg} / \mathrm{mL}$. Cells with materials were further incubated in anaerobic conditions for $24 \mathrm{~h}$, and $150 \mu \mathrm{L}$ of MTT solution $(0.5 \mathrm{mg} / \mathrm{mL})$ was then added to each well. After $4 \mathrm{~h}$ of treatment, the MTT solution was discarded, and $150 \mu \mathrm{L}$ of dimethyl sulfoxide (DMSO) was added to dissolve crystals. Finally, the absorbance was measured at $490 \mathrm{~nm}$ using a microplate reader (Synergy 2, BioTek, USA). (2) B16-F10 cells were incubated in 96-well plates and cultured for $24 \mathrm{~h}$ under anaerobic conditions. The cells were divided into three groups: control, HMSNs-Ce6@CPPO-PFC@C, and HMSNs-GOx@CPPO-PFC@C groups. The concentration of the nanoparticles was $0.2 \mathrm{mg} / \mathrm{mL}$. Cells with materials were further incubated in anaerobic conditions for $24 \mathrm{~h}$, and $150 \mu \mathrm{L}$ of MTT solution $(0.5 \mathrm{mg} / \mathrm{mL})$ was then added to each well. After $4 \mathrm{~h}$ of treatment, the MTT solution was discarded, and $150 \mu \mathrm{L}$ of DMSO was added to dissolve crystals. Finally, the absorbance was measured at $490 \mathrm{~nm}$ using a microplate reader (Synergy 2, BioTek, USA).

Western blot. Cells were lysed in a radioimmunoprecipitation assay buffer containing $20 \mathrm{mM}$ Tris (pH 7.5), $150 \mathrm{mM} \mathrm{NaCl}, 50 \mathrm{mM} \mathrm{NaF}, 1 \%$ nonidet P- $40,0.1 \%$ deoxycholic acid, $0.1 \%$ sodium dodecyl sulfate (SDS), $1 \mathrm{mM}$ EDTA, $1 \mathrm{mM}$ phenylmethylsulfonyl fluoride, and $1 \mu \mathrm{g} / \mathrm{mL}$ leupeptin. Proteins were resolved by SDS-polyacrylamide gel electrophoresis $(10 \%)$ and transferred onto a polyvinylidene fluoride membrane. The membranes were blocked with $5 \%$ fat-free dry milk and incubated with primary antibody overnight at $4{ }^{\circ} \mathrm{C}$. The membranes were incubated with horseradish peroxidase-conjugated secondary antibodies. Specific proteins were visualized with enhanced chemiluminescence detection reagent (Santa Cruz Biotechnology, Inc.). The blots were analyzed using a Bio-Rad imaging system (Bio-Rad, Hercules, CA, USA).

In vivo targeting and pharmacokinetics by ICP-AES. All animal experiments were conducted and agreed with the Principles of Laboratory Animal Care (People's Republic of China). BalB/C mice (4-6 weeks old, female, $\sim 20 \mathrm{~g}$ ) were fed with normal conditions of $12 \mathrm{~h}$ light and dark cycles and given access to food and water ad libitum. 
$\mathrm{BalB} / \mathrm{C}$ mice with lung metastatic tumors were injected intravenously with HMSNs-Ce6@C or HMSNs-Ce6 (40 mg/kg). For the targeting experiment, at $24 \mathrm{~h}$ post injection, the mice were killed and dissected for imaging. Before the ICP-AES experiment, the metastatic tumors were separated from normal lung tissue by cutting the black B16-F10 metastatic tumors from the total lung tissue. Then, the metastatic tumors and five major organs were dissolved in aqua regia $\left(\mathrm{HCl}: \mathrm{HNO}_{3}\right.$ : $\mathrm{HClO}_{4}=3: 1: 2$, v:v:v) for ICP-AES analysis of Si. For pharmacokinetics, the excrement of the mice was collected at different post-injection times $(1,2,4,8,12$, 24,48 , and $72 \mathrm{~h}$ ) and dissolved in aqua regia $\left(\mathrm{HCl}: \mathrm{HNO}_{3}: \mathrm{HClO}_{4}=3: 1: 2\right.$, v:v:v) for ICP-AES analysis of $\mathrm{Si}$.

In vivo therapeutic effect of the bio-NRs. BalB/C mice with lung metastatic tumors were divided into five groups and injected intravenously with PBS, HMSNs-Ce6@CPPO-PFC/O $2 @ C, H M S N s-G O x @ C P P O-P F C / O_{2} @ C, H M S N s-$ GOx-Ce6@CPPO-PFC@C, or HMSNs-GOx-Ce6@CPPO-PFC/O ${ }_{2} @ C$. The dose was $40 \mathrm{mg} / \mathrm{kg}$. The mice were killed and dissected at 14 days post injection to assess the therapeutic effect of the nanoparticles. The black B16-F10 metastatic tumors were cut off from the total lung tissue to the maximum extent. Subsequently, the mass of metastatic tumors and the normal lung was then measured using an analytical balance. The mass percentage of metastases was calculated according to the weight of metastatic tumors/weight of normal lung. The body weights of the mice were recorded every other day during 14 days of therapy, and the survival rate was calculated for 30 days. Furthermore, the heart, liver, spleen, lung, and kidney of mice were used for histological sectioning and H\&E staining.

\section{Data availability}

All relevant data that support the findings of this study are available from the corresponding author upon reasonable request.

Received: 27 June 2018 Accepted: 17 October 2018

Published online: 28 November 2018

\section{References}

1. Chaffer, C. L. \& Weinberg, R. A. A perspective on cancer cell metastasis. Science 331, 1559-1564 (2011).

2. Valastyan, S. \& Weinberg, R. A. Tumor metastasis: molecular insights and evolving paradigms. Cell 147, 275-292 (2011).

3. Detty, M. R., Gibson, S. L. \& Wagner, S. J. Current clinical and preclinical photosensitizers for use in photodynamic therapy. J. Med. Chem. 47, 3897-3915 (2004).

4. Huang, L. et al. Enhancing photodynamic therapy through resonance energy transfer constructed near-infrared photosensitized nanoparticles. Adv. Mater. 29, 1604789 (2017).

5. Cheng, L. et al. Functional nanomaterials for phototherapies of cancer. Chem. Rev. 114, 10869-10939 (2014).

6. Lucky, S. S., Soo, K. C. \& Zhang, Y. Nanoparticles in photodynamic therapy. Chem. Rev. 115, 1990-2042 (2015).

7. Fan, W., Huang, P. \& Chen, X. Overcoming the Achilles' heel of photodynamic therapy. Chem. Soc. Rev. 45, 6488-6519 (2016).

8. Zhou, Z., Song, J., Nie, L. \& Chen, X. Reactive oxygen species generating systems meeting challenges of photodynamic cancer therapy. Chem. Soc. Rev. 45, 6597-6626 (2016).

9. Zhou, B., Shi, B., Jin, Y. \& Liu, X. Controlling upconversion nanocrystals for emerging applications. Nat. Nanotechnol. 10, 924-936 (2015).

10. Kachynski, A. V. et al. Photodynamic therapy by in situ nonlinear photon conversion. Nat. Photonics 8, 455-461 (2014).

11. Teo, R. D., Termini, J. \& Gray, H. B. Lanthanides: applications in cancer diagnosis and therapy. J. Med. Chem. 59, 6012-6024 (2016).

12. Xiao, Q. et al. A core/satellite multifunctional nanotheranostic for in vivo imaging and tumor eradication by radiation/photothermal synergistic therapy. J. Am. Chem. Soc. 135, 13041-13048 (2013).

13. Cui, S. et al. In vivo targeted deep-tissue photodynamic therapy based on near-infrared light triggered upconversion nanoconstruct. ACS Nano 7 , 676-688 (2013).

14. Shuhendler, A. J. et al. Real-time imaging of oxidative and nitrosative stress in the liver of live animals for drug-toxicity testing. Nat. Biotechnol. 32, 373-380 (2014).

15. Li, P. et al. A new polymer nanoprobe based on chemiluminescence resonance energy transfer for ultrasensitive imaging of intrinsic superoxide anion in mice. J. Am. Chem. Soc. 138, 2893-2896 (2016).

16. Mao, D. et al. Chemiluminescence-guided cancer therapy using a chemiexcited photosensitizer. Chem 3, 991-1007 (2017).

17. Lee, D. et al. In vivo imaging of hydrogen peroxide with chemiluminescent nanoparticles. Nat. Mater. 6, 765-769 (2007).
18. Stone, J. R. \& Yang, S. Hydrogen peroxide: a signaling messenger. Antioxid. Redox Signal. 8, 243-270 (2006).

19. Zhou, J. et al. Engineering of a nanosized biocatalyst for combined tumor starvation and low-temperature photothermal therapy. ACS Nano 12, 2858-2872 (2018)

20. Fan, W. et al. Glucose-responsive sequential generation of hydrogen peroxide and nitric oxide for synergistic cancer starving-like/gas therapy. Angew. Chem. Int. Ed. 56, 1229-1233 (2016).

21. Liu, L. et al. An electrochemical biosensor with dual signal outputs: toward simultaneous quantification of $\mathrm{pH}$ and $\mathrm{O} 2$ in the brain upon ischemia and in a tumor during cancer starvation therapy. Angew. Chem. Int. Ed. 56, 10471-10475 (2017).

22. Sun, L. et al. Real-time imaging of single-molecule enzyme cascade using a DNA origami raft. J. Am. Chem. Soc. 139, 17525-17532 (2017).

23. Gross, A. J. et al. Redox-active glyconanoparticles as electron shuttles for mediated electron transfer with bilirubin oxidase in solution. J. Am. Chem. Soc. 139, 16076-16079 (2017)

24. Zheng, X. et al. Tracking cancer metastasis in vivo by using an iridiumbased hypoxia-activated optical oxygen nanosensor. Angew. Chem. Int. Ed. 54 8094-8099 (2015).

25. Vaupel, P. \& Mayer, A. Hypoxia and cancer: significance and impact on clinical outcome. Cancer Metastas. Rev. 26, 225-239 (2007).

26. Denko, N. C. Hypoxia, HIF1 and glucose metabolism in the solid tumor. Nat. Rev. Cancer 8, 705-713 (2008).

27. $\mathrm{Yu}, \mathrm{Z}$. et al. A pre-protective strategy for precise tumor targeting and efficient photodynamic therapy with a switchable DNA/upconversion nanocomposite. Chem. Sci. 9, 3563-3569 (2018).

28. Bottini, M., Rosato, N. \& Bottini, N. PEG-modified carbon nanotubes in biomedicine: current status and challenges ahead. Biomacromolecules 12, 3381-3393 (2011).

29. Kobayashi, H., Turkbey, B., Watanabe, R. \& Choyke, P. L. Cancer drug delivery: considerations in the rational design of nanosized bioconjugates. Bioconjugate Chem. 25, 2093-2100 (2014).

30. Lao, Y.-H., Phua, K. K. L. \& Leong, K. W. Aptamer nanomedicine for cancer therapeutics: barriers and potential for translation. ACS Nano 9, 2235-2254 (2015).

31. Srinivasarao, M., Galliford, C. V. \& Low, P. S. Principles in the design of ligand-targeted cancer therapeutics and imaging agents. Nat. Rev. Drug. Discov. 14, 203-219 (2015).

32. Ballou, B. et al. Sentinel lymph node imaging using quantum dots in mouse tumor models. Bioconjugate Chem. 18, 389-396 (2013).

33. Peer, D. et al. Nanocarriers as an emerging platform for cancer therapy. Nat. Nanotechnol. 2, 751-760 (2007).

34. Li, S.-Y. et al. Cancer cell membrane camouflaged cascade bioreactor for cancer targeted starvation and photodynamic therapy. ACS Nano 11, 7006-7018 (2017).

35. Sun, H. et al. Cancer-cell-biomimetic nanoparticles for targeted therapy of homotypic tumors. Adv. Mater. 28, 9581-9588 (2016).

36. Zou, W. Immunosuppressive networks in the tumour environment and their therapeutic relevance. Nat. Rev. Cancer 5, 263-274 (2005).

37. Rodriguez, P. L. et al. Minimal "self" peptides that inhibit phagocytic clearance and enhance delivery of nanoparticles. Science 339, 971-975 (2013).

38. Chen, D., Li, L., Tang, F. \& Qi, S. Facile and scalable synthesis of tailored silica "nanorattle" structures. Adv. Mater. 21, 3804-3807 (2009).

39. $\mathrm{Xu}, \mathrm{K}$. et al. A near-infrared reversible fluorescent probe for real-time imaging of redox status changes in vivo. Chem. Sci. 4, 1079-1086 (2013).

40. Li, Y. et al. Constructing solid-gas-interfacial fenton reaction over alkalinized$\mathrm{C}_{3} \mathrm{~N}_{4}$ photocatalyst to achieve apparent quantum yield of $49 \%$ at $420 \mathrm{~nm}$. J. Am. Chem. Soc. 138, 13289-13297 (2016).

41. He, W. et al. Photogenerated charge carriers and reactive oxygen species in $\mathrm{ZnO} / \mathrm{Au}$ hybrid nanostructures with enhanced photocatalytic and antibacterial activity. J. Am. Chem. Soc. 136, 750-757 (2014).

42. Semenza, G. L. Oxygen sensing, hypoxia-inducible factors, and disease pathophysiology. Annu. Rev. Pathol. 9, 47-71 (2014).

43. Zhao, Q. et al. Interaction between circulating galectin-3 and cancerassociated MUC1 enhances tumour cell homotypic aggregation and prevents anoikis. Mol. Cancer 9, 154-166 (2010).

44. Majeti, R. et al. CD47 is an adverse prognostic factor and therapeutic antibody target on human acute myeloid leukemia stem cells. Cell 138, 286-299 (2009).

45. Naor, D., Sionov, R. V. \& Ish-Shalom, D. CD44: structure, function, and association with the malignant process. Adv. Cancer Res. 71, 241-319 (1997).

46. $\mathrm{Wu}, \mathrm{W}$. et al. TLR ligand induced IL- 6 counter-regulates the anti-viral CD8 ${ }^{+}$ $\mathrm{T}$ cell response during an acute retrovirus infection. Sci. Rep. 5, 10501-10514 (2015).

47. Trinchieri, G. Interleukin-12 and the regulation of innate resistance and adaptive immunity. Nat. Rev. Immunol. 3, 133-146 (2003). 


\section{Acknowledgements}

This work was supported by the National Natural Science Foundation of China $(21535004,91753111,21874086,21775094,21505087,21390411)$ and the Key Research and Development Program of Shandong Province (2018YFJH0502).

\section{Author contributions}

Z.Y., N.L., and B.T. conceived and designed the experiments. Z.Y. and P.Z. performed the experiments. Z.Y., W.P., N.L., and B.T. analyzed the data. Z.Y. and W.P. contributed the schematic materials. Z.Y., N.L., and B.T. co-wrote the paper. Z.Y., W.P., and N.L. edited the manuscript.

\section{Additional information}

Supplementary Information accompanies this paper at https://doi.org/10.1038/s41467018-07197-8.

Competing interests: The authors declare no competing interests.

Reprints and permission information is available online at http://npg.nature.com/ reprintsandpermissions/
Publisher's note: Springer Nature remains neutral with regard to jurisdictional claims in published maps and institutional affiliations.

\section{(c) (1)}

Open Access This article is licensed under a Creative Commons Attribution 4.0 International License, which permits use, sharing, adaptation, distribution and reproduction in any medium or format, as long as you give appropriate credit to the original author(s) and the source, provide a link to the Creative Commons license, and indicate if changes were made. The images or other third party material in this article are included in the article's Creative Commons license, unless indicated otherwise in a credit line to the material. If material is not included in the article's Creative Commons license and your intended use is not permitted by statutory regulation or exceeds the permitted use, you will need to obtain permission directly from the copyright holder. To view a copy of this license, visit http://creativecommons.org/ licenses/by/4.0/.

(c) The Author(s) 2018 